\title{
Interferometric aperture synthesis for next generation passive millimetre wave imagers
}

\author{
Neil A. Salmon*, Peter Wilkinson, Chris Taylor \\ Jodrell Bank Centre for Astrophysics, University of Manchester, Manchester, M13 9PL, UK
}

\begin{abstract}
This paper discusses the phase effects in the near-field associated with aperture synthesis imaging. The results explain why in some regions of the near-field it is possible to use Fourier transform techniques on a visibility function to create images. However, to generate images deep inside the near-field alternative processing techniques such as the G-matrix method are required. Algorithms based on this technique are used to process imagery from a proof of concept $22 \mathrm{GHz}$ aperture synthesis imager ${ }^{[1]}$. Techniques for generating synthetic cross-correlations for the aperture synthesis technique are introduced and these are then validated using the image creation algorithms and real data from the proof of concept imager. Using these data the phenomenon of aliasing is explored. The simulation code is then used to illustrate how the effects of aliasing may be minimised by randomising the locations of the antennas over the aperture. The simulation tool is used to show how in the near field the technique can provide a range resolution in 3D imaging of a couple of millimetres when operating with a wavelength of $13 \mathrm{~mm}$. Moving to illustrate the quality of images generated by a next generation aperture synthesis imagers, the software is extended to systems with hundreds of receiver channels.
\end{abstract}

Keywords: Aperture synthesis, imaging, digital beam-forming, security screening, aliasing, passive, simulation

\section{INTRODUCTION}

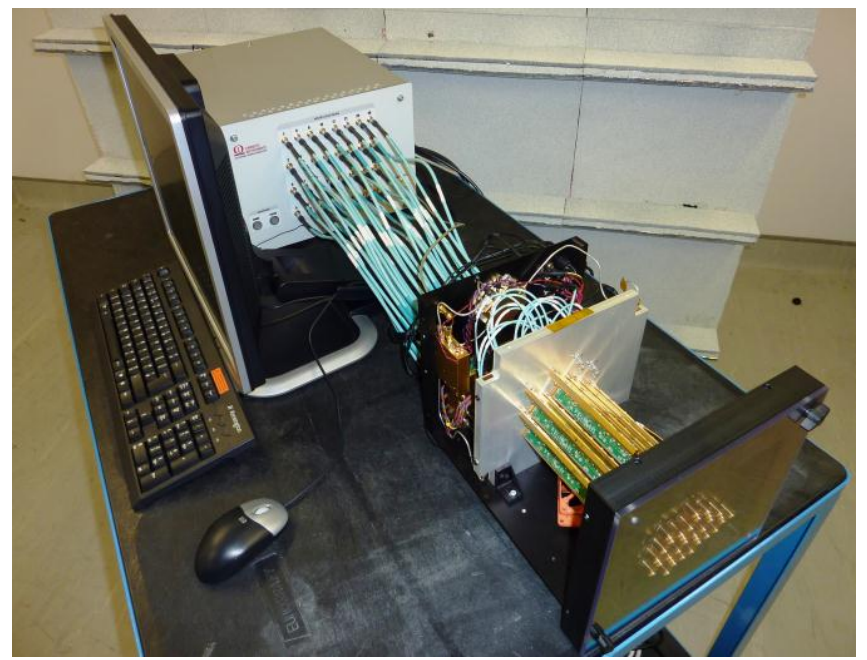

Figure 1. The 32-channel DTI aperture synthesis imager showing the $22.51 \mathrm{GHz}$ RF (300 MHz bandwidth) antenna-receiver (bottom right) outputting a $3.71 \mathrm{GHz} \mathrm{IF}$ via cables to the cross-correlator (top left). The system sits on top of a portable laboratory bench ${ }^{[1]}$.
The evolution of passive millimetre wave imaging sensors is taking many years and systems are still not satisfying market expectations. The two essential problems are imager cost (which influences performance) and size. Although the fundamental capabilities of millimetre wave imagers are proven for the markets of security screening of personnel and all-weather imaging, when implementing systems the high costs of receivers restrict the numbers of sensing elements and this compromises the performance.

Imager size is the other major obstacle in the evolution of millimetre wave systems. For the security screening, systems are required to fit into the confined spaces around entrances, and walls and doors in these scenarios are opportune surfaces for deployment. Similarly, for allweather sensors, aircraft fuselages and wings are ideal surfaces for deployment. However, the volumes of quasioptical imagers are incompatible with such deployments, as imager apertures need to be upward of half a metre in size to achieve the required image resolution. Unfortunately systems with smaller aperture sizes fail to achieve the necessary spatial resolution. Furthermore, this situation has been helped little by moving to the higher frequencies (> $200 \mathrm{GHz}$ ), with the promises of better resolutions and reduced imager volumes, as devices in these bands generally have lower performances and are much more expensive.

\footnotetext{
* Correspondence: Email: neil.salmon at mmw-sensors.com; Telephone: +44 7921172892
} 
Aperture synthesis offers an attractive but unconventional imaging alternative for security and aerospace applications, as the sensor lends itself to deployment in a planar format over surfaces. The image is formed in a digital processor, so no bulky optical imaging components are required. The derisking of the aperture synthesis imaging approach has been carried out using a number of complementary systems, in the near and far-field, for wide and narrow field of view imaging, and at a number of frequencies, namely $12,22,94,183 \mathrm{GHz}{ }^{[1][2][3]}$. Assessment of system performance is all in accordance with the accepted theory of aperture synthesis imaging from radio astronomy and simulations. The system most recently demonstrated is the DTI aperture synthesis imager at $22 \mathrm{GHz}$ and this delivered real-time video rate (25 Frames/sec) imagery of indoor and outdoor subjects and also high-speed video (200 Frames/sec) and is illustrated in Figure $1^{[1]}$.

This paper discusses the most recent work in this area including the examination of near-field phase effects, processing of experimental data into imagery using Fourier transforms and the G-matrix technique, generation of synthetic crosscorrelation data and model validation using real data, and simulations for a next phase demonstrator imager.

\section{MODELLING PHASE EFFECTS IN THE NEAR FIELD}

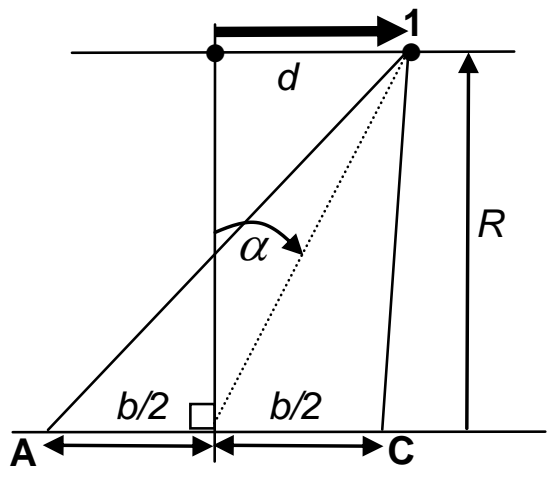

Figure 2: The geometry associated with the near field phase shifts measured on a baseline of length $b$ (A-C) due to a lateral source displacement, $d$, from the centre to position 1 .

In the near field region of an antenna the curvature of wave-fronts needs to be taken into consideration if the phases of crosscorrelations are to be processed into imagery. The phase deviation across an aperture of size $D$ between a curved wave-front from a source and a plane wave becomes greater than $\pi / 8$ for sources closer than the Rayleigh distance, given by Eq. 1, where $\lambda$ is the wavelength. As the phase of the wave plays a key role in imaging, it is useful to examine the phase shift on the cross-correlation for a baseline as a source moves perpendicularly away from the centre of the field of view. It is this phase shift and how it varies between the near and far field which determines how well the classic far-field aperture synthesis techniques using Fourier transforms of visibility functions can be used in the near field.

The far-field phase as the source moves perpendicularly outwards from the centre of the field of view is given by Eq. 2, where $b$ is the baseline and $\alpha$ is the angle from the normal to the baseline as illustrated in Figure 2. Given the geometry, the true phase of the cross-correlation is given by Eq. 3, where the lengths are given by

Eq. 4 and 5, and $d$ is the displacement away from the normal and $R$ is the range as related in Eq. 6. The difference between the true phase and the far-field phase as the source moves away from the centre is given by Eq. 7 . This is plotted as a function of displacement in Figure 3 for a range of $0.5 \mathrm{~m}$ and baseline lengths of 10,20 and $50 \mathrm{~cm}$.

$$
\begin{aligned}
& R_{\text {Rayleigh }}=\frac{2 D^{2}}{\lambda} \quad \text { (1) } \quad \phi_{R \rightarrow \infty}=2 \pi\left(\frac{b}{\lambda}\right) \sin \alpha \quad \text { (2) } \quad \phi=2 \pi \frac{\left(l_{A 1}-l_{C 1}\right)}{\lambda} \\
& l_{A 1}^{2}=\left(\frac{b}{2}\right)^{2}+\left(\frac{R}{\cos \alpha}\right)^{2}+b R \tan \alpha \\
& \frac{d}{R}=\tan \alpha \\
& l_{C 1}^{2}=\left(\frac{b}{2}\right)^{2}+\left(\frac{R}{\cos \alpha}\right)^{2}-b R \tan \alpha \\
& \Delta \phi=\phi-\phi_{R \rightarrow \infty}
\end{aligned}
$$

It can be seen from Figure 3 that for a baseline length of $\sim 10 \mathrm{~cm}$, the maximum deviation in the phase is only about $3^{\circ}$, even though the Rayleigh distance of $1.5 \mathrm{~m}$ indicates it is well inside the near-field regime. This means that an imaging system with an aperture of $10 \mathrm{~cm}$ could effectively use the Fourier transform of a visibility function to create images, and experimentation has shown this to be the case ${ }^{[1]}$. However, it can also be appreciated from the figure that for a baseline of $50 \mathrm{~cm}$ the phase deviation, at several hundred degrees, is much greater. Such a system could correspond to a 
portal imaging system. This large phase deviation would be incompatible with the Fourier transform method to create images.
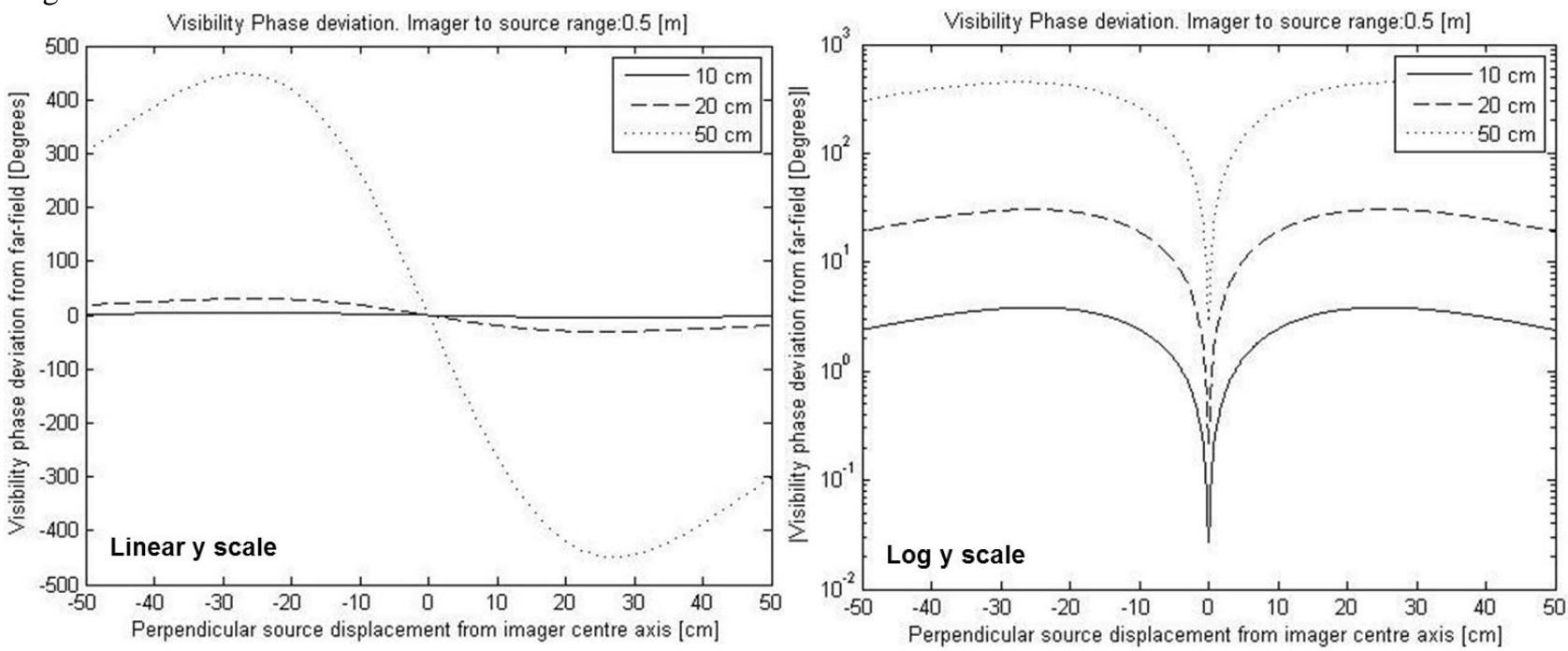

Figure 3: The difference between the actual and far-field phase deviations for a source as it is moved from the centre of the field of view outwards from a normal to the baseline. The sign of the phase difference is shown on a linear $y$ scale graph (left) and the magnitude is shown on the log y scale graph (right). These indicate the greatest change in phase occurs in the centre of the field of view. The legend indicates the base-line lengths of $10 \mathrm{~cm}, 20 \mathrm{~cm}$ and $50 \mathrm{~cm}$.

\section{PROCESSING IMAGERY USING THE G-MATRIX AND THE FOURIER TRANSFORM}

\subsection{Generating simulated cross-correlations}

The cross-correlation from a source in the scene is now investigated to understand how images can be created from a

Antenna

Aperture array

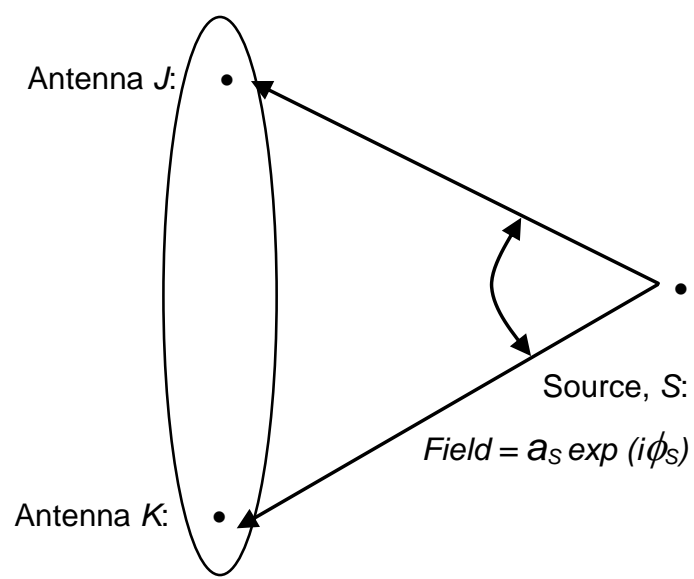

Figure 4: The electric field from a source in the field of view is measured at pairs of antennas $J, K$ in the aperture of the imager as a cross-correlation whole series of cross-correlations and how simulations of synthetic cross-correlations may be created for the purpose of simulation. If the source, $S$, lying within the field of view of the imager has an electric field amplitude $a_{S}$ and a random phase $\phi_{S}$, the voltage at an antenna $J$, at a location $r_{S}$, will be given by Eq. 8. As the voltage at this antenna location is the sum of voltages from all sources in the field of view, the total voltage is given by Eq. 9. As the cross-correlation process is given by Eq. 10, substitution of Eq. 8 and 9 gives Eq. 11 for the cross-correlation for all sources in the scene. As the phases $\phi_{S}$ are random and there are many sources in the field of view, over a long period of time the cross-terms in Eq. 11 vanish and the cross-correlation reduces to that given by Eq. 12. This is the basis for the generating the synthetic crosscorrelations of the forward simulations.

$$
\begin{gathered}
v_{S, J}=\frac{g_{S, J} a_{S, J}}{r_{S, J}} \exp j\left(\phi_{S}+\phi_{J}\right) \\
v_{J}=\sum_{S} v_{S, J}
\end{gathered}
$$




$$
V_{J, K}=\left\langle v_{J} v_{K}^{*}\right\rangle
$$

$$
V_{J, K}=\left\langle\left(\sum_{S} \frac{g_{S, J} a_{S, J}}{r_{S, J}} \exp i\left(\phi_{S}+\phi_{J}\right)\right)\left(\sum_{S} \frac{g_{S, K} a_{S, K}}{r_{S, K}} \exp i\left(\phi_{S}+\phi_{K}\right)\right)^{*}\right\rangle
$$

\subsection{Inverting cross-correlations into images}

The general case of the relationship between the cross-correlations and the temperature distribution is given by Eq. 12 and written as a matrix equation this can be inverted to give the temperature $T$, and this technique is referred to as the Gmatrix technique ${ }^{[6]}$, where $\mathrm{G}+$ is the pseudo inverse of the matrix representing the response of the cross-correlations to the distribution of temperature sources in the scene (ie the image). In this approach, the data in both the crosscorrelations and the image are stored as vectors. In the creation of the G-matrix there is a mapping of the sources in two dimensions to the data in a vector representation. After inversion the resulting temperatures stored as a vector must be unpacked by the reverse mapping to create the two dimensional image. In the specific case of the far-field and where the antenna gain is constant over the source, the gain and ranges come out of the summation and the cross-correlation can be written as Eq. $14^{[4]}$. Written in matrix form the source temperature can be calculated by taking the Fourier transform of the cross-correlations, as in Eq. 15, and this is the basis of radio astronomy aperture synthesis technique ${ }^{[5]}$.

$$
\begin{array}{r}
V_{J, K}=\sum_{S} \frac{g_{S, J} g_{S, K} T_{S}}{r_{S, J} r_{S, K}} \exp i\left(\phi_{J}-\phi_{K}\right) \\
V_{J, K}=\frac{g^{2}}{r^{2}} \sum_{S} T_{S} \exp i\left(\phi_{J}-\phi_{K}\right)
\end{array}
$$

$$
\begin{aligned}
& T=G^{+} V \\
& T=F T^{-1} V
\end{aligned}
$$

Images created using the G-matrix and the Fourier transform technique from above and using real data taken from the DTI imager are shown in Figure 5. They show an urban scene and illustrate cold sky, warm buildings, and people and vehicles can be recognised moving around. In the G-matrix technique, the phases of the visibilities are calibrated using a single measurement from a noise source in the centre of the field of view. The magnitudes of the individual gains of the antennas are calculated using an empirical fit to measured antenna patterns and an accurate (to within $0.45 \mathrm{~mm}$ ) physical measurement of the locations of the antennas is used. The assumption here is that the phase centres of the antennas geometrically all have the same locations in space with respect to the antenna waveguide centres and that any phase differences between this and sources are based purely on the geometrical distance. A better option would be to measure the positions of the phase centres of the antennas using radiation at the probe frequency of $22 \mathrm{GHz}$. Off-axis phase performance from the antennas is something still to be accounted for, and its absence in this processing may be responsible for errors in the image.

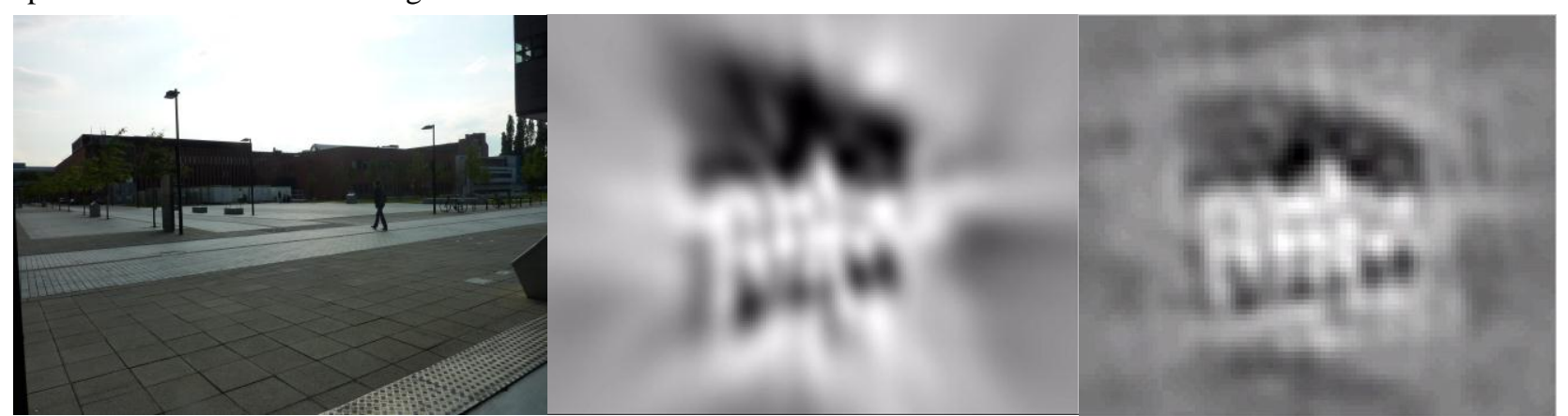

Figure 5: Images taken of an urban scene (left) using the DTI proof of concept aperture synthesis imager ${ }^{[1]}$ and processed using the G-matrix (centre) and Fourier transform (right) techniques

The absence of antenna phase in this implementation of the G-matrix technique and assumptions about the positions of the antenna phase centres may be rectified in a brute force way by measuring cross-correlations at every pixel in the image using the noise source. This however requires a large number of measurements for images with many pixels. There may be more innovative and resourceful techniques to realising a G-matrix calibration by making a smaller set of 
measurements across the full field of view and making assumptions about the similarity of beam-patterns from the antennas.

A third option as an alternative to the G-matrix would be sought, as the complexity of a 2D Fourier transform using an FFT of $m$ cross-correlations is proportional to $\operatorname{mLog}_{2} m$ whereas the complexity of the G-matrix multiplication is proportional to $m x p$, where $p$ is the number of pixels and $p \geq m$. The G-matrix technique is not therefore particularly efficient. One possible solution might be to split the image into a mosaic of smaller local regions where the Fourier transform would be used to create the image in each.

\section{IMAGE SIMULATION}

\subsection{Simulation Validation}

The experimental data generated by the DTI aperture synthesis imager is ideal for validating the software used to simulate the generation of synthetic cross-correlations discussed in the last section. One of the key findings from experimentation with the DTI imager is that the system is sensitive to aliasing. Understanding this will be fundamental in the design of follow-on imaging systems.

Experiments in aliasing were conducted in which a lamp acting as a point source was moved from the centre of the imager field of view towards the right, and also from the centre to the top of the field of view, so as to force the creation
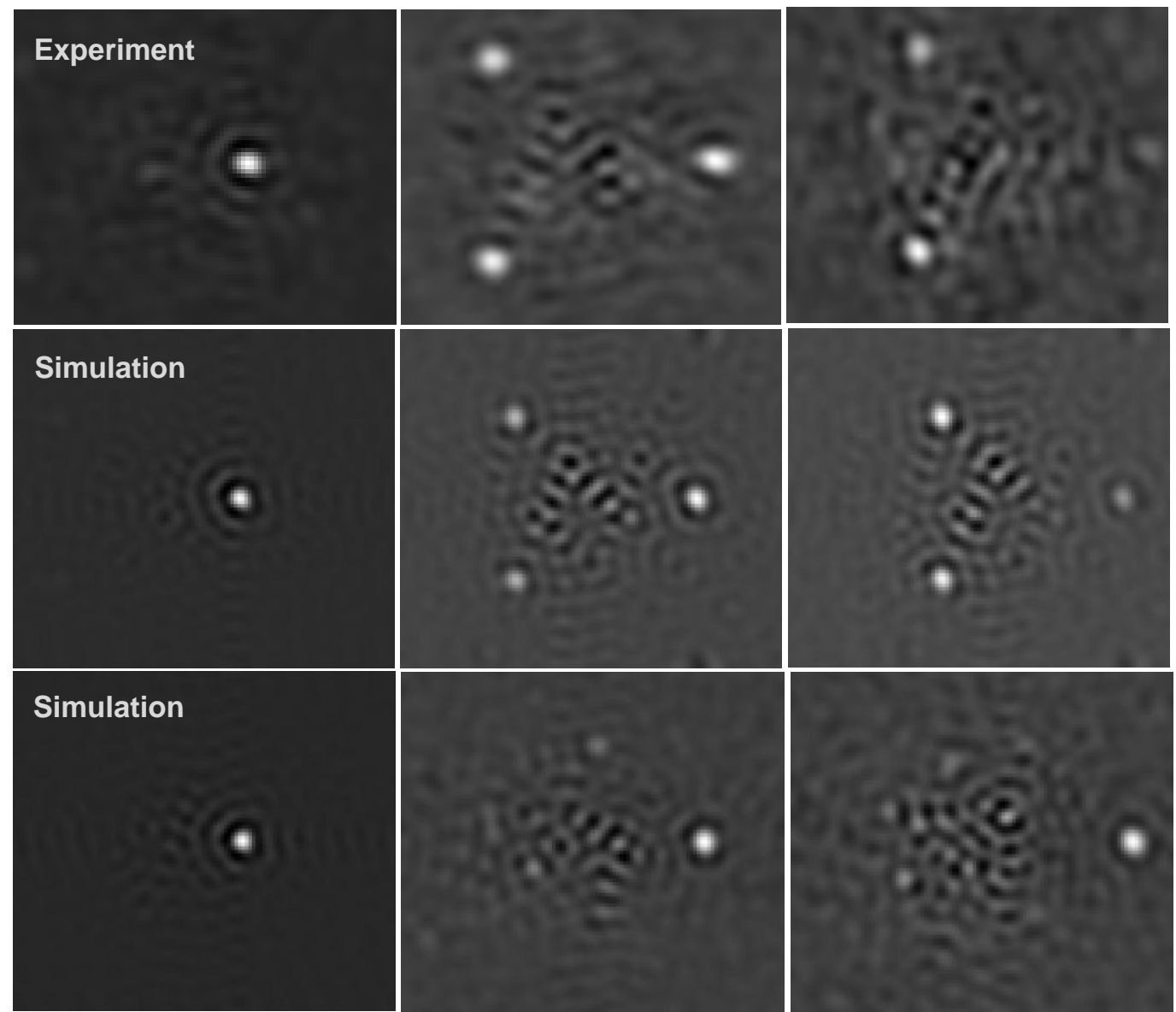

Figure: 6 Top - Images generated using the Fourier transform technique to process cross-correlations measured using the DTI aperture synthesis imager of a lamp moving from the centre of the field of view to the right. Centre - Images generated using the same technique but processing synthetically generated cross-correlations. Bottom - Images generated using the same technique from synthetic cross-correlations but with a fully randomised array of antennas. 
of aliases. These images are shown in the top images of Figures 6 and 7. It can be seen from Figure 6 that when the source is moved from the centre to the right, two things happen. Firstly, the reproduction of the lamp in its true position becomes fainter and secondly two artefacts appear above and below the image centre on the left hand side. These two artefacts are aliases and are generated as the wave-front sampling across the array is at less than the Nyquist spatial frequency. Using Eq. 12 synthetic cross-correlations were generated and ran through the imaging algorithm to generate the images in the centre of these figures. This exact same phenomenon is reproduced, with the conclusion that the simulation is correctly reproducing the measured effects of aliasing.

When the source is moved upward from the centre of the field of view there is a similar phenomenon illustrated in Figure 6. Measurements show the reproduction of the lamp becomes fainter and a bright spot appears to come in from the bottom of the image, and this is the alias. The simulation using the synthetic cross-correlations in the centre of the figure reproduces the exact measured phenomenon.

Since this simulation tool has been validated for the reproduction of aliases, it is useful to exploit this to investigate the effect of randomisation of the antenna positions on the reproduction of aliases. The DTI imager antenna array geometry
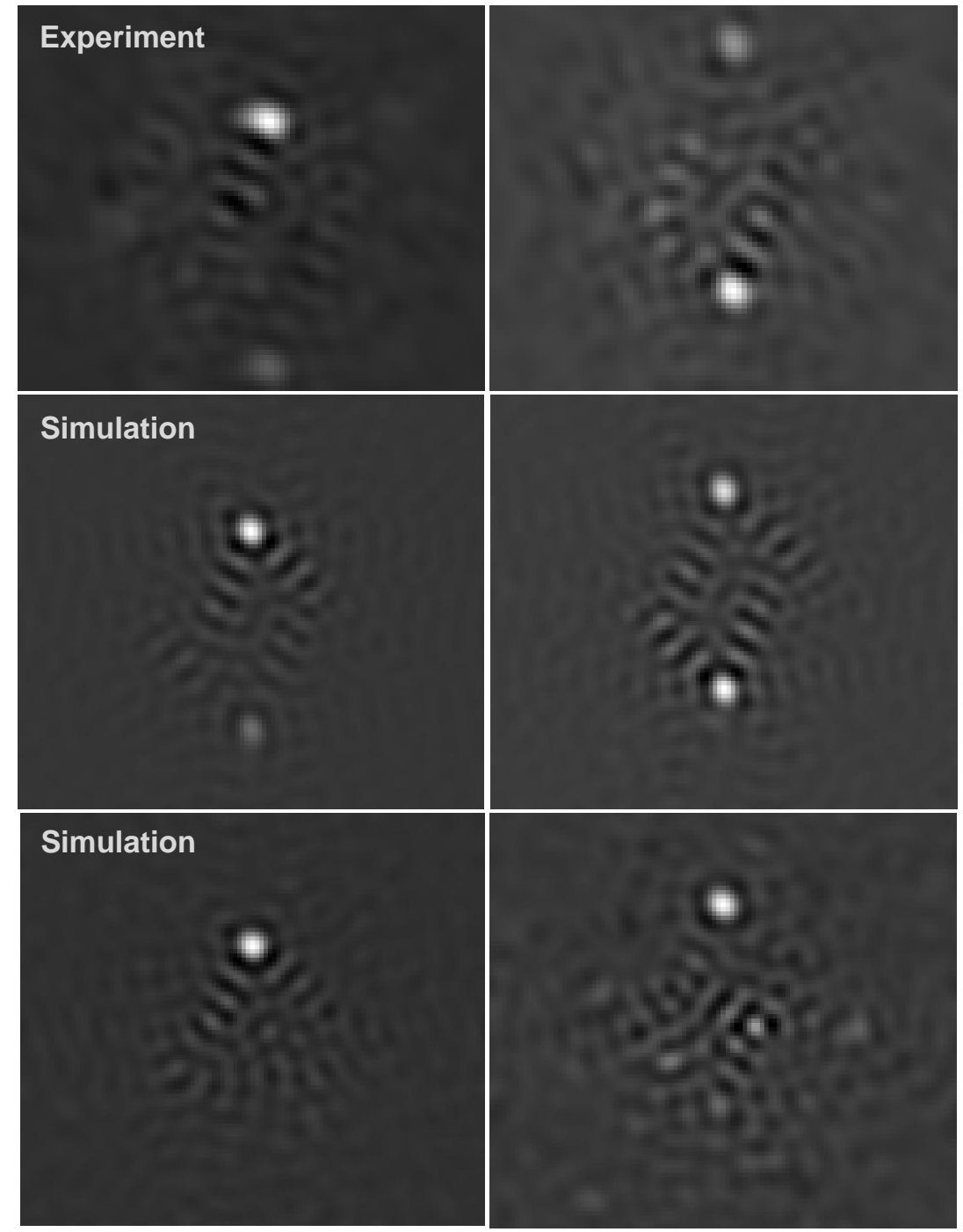

Figure 7: Images from; Top (experiment); Centre (simulation), Bottom (simulation with random array), for a point source moving vertically upwards from the centre of the field of view. was taken and the locations of the antennas were fully randomised. The same simulation was performed whereby the lamp was moved to the right and upwards and the results of this simulation are shown in Figures 6 and 7 at the bottom. Two observations can be made, firstly the reproduction of the lamp appears strong and secondly the bright alias spot disappears. In fact it looks as if the energy in the alias has been distributed in other parts of the image.

The conclusion about the randomisation of the array is that it strengthens the reproduction of the sources in the image and weakens the effects of aliasing. This is almost like saying that random sampling is in some way superior to the regular sampling associated with the Nyquist sampling theorem.

\subsection{Effects of aliasing in simulated images}

Further aliasing effects and their removal by randomising the array can be demonstrated by simulation of the DTI aperture synthesis imager array. For the purpose of simulating these effects in security screening, the Vitruvian man was taken as subject. For this configuration the synthetic crosscorrelations were calculated using Eq. 12. The Fourier transform technique was then used to reproduce 
the image from the synthetic cross-correlations, and this image is shown in Figure 8. The DTI imager array was then taken and the antenna positions were fully randomised and the resulting array is shown in Figure 8 . In this process each antenna was taken in turn and its position randomised by an amount equal to the hexagonal grid constant of the original antenna layout. This was done on each antenna in such a way as to avoid collisions between antennas. This means that such an array could actually be built in practice. Using the fully randomised array, synthetic cross-correlations were calculated and then run through the Fourier transform imaging algorithm and the image which resulted is shown in Figure 8. It can be appreciated from these images that the main effect of the randomisation is in the reduction of blob structures around the periphery of the image. In comparisons to images in the previous section these blobs are interpreted as aliases. It appears that the randomisation of the antenna array removes the aliases from the image.
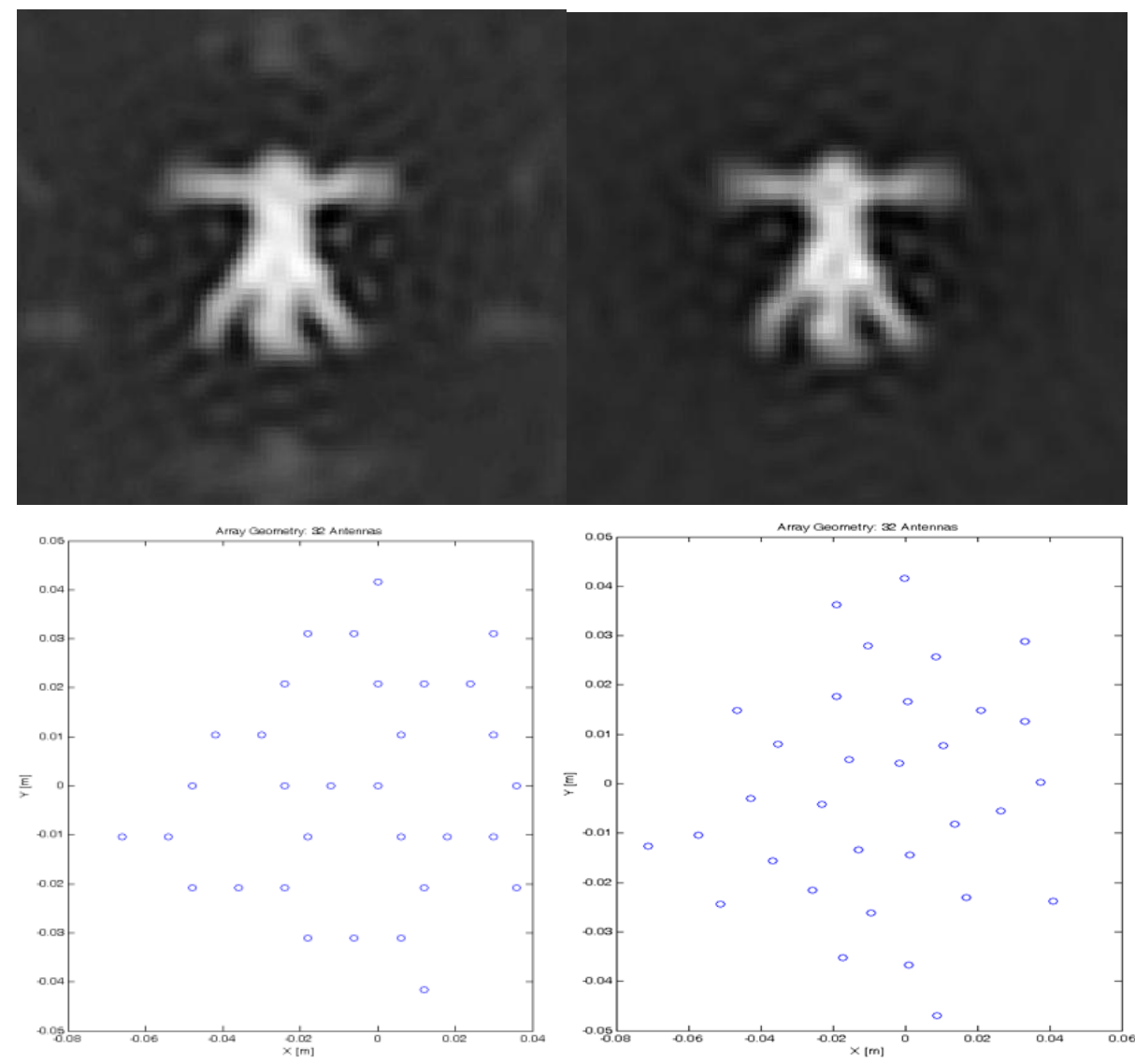

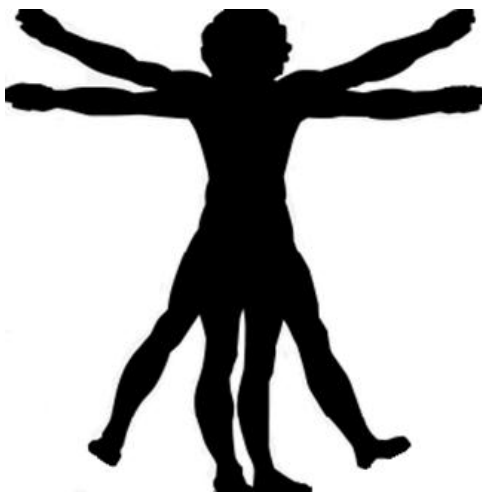

Figure 8: Image simulation of the Vitruvian man (right-above) showing the effect of aliasing from the DTI imager (left) as blobs of higher intensity around the image periphery. The effect of full randomisation of the antenna array is to remove the aliases from the image (centretop).

\subsection{Resolution in passive ranging}

Operating in the near-field has potential for passive ranging. This is because the wave-fronts are curved and measurement of a minimum of three points on a curve can provide information about the origin of the wave-front. In the case of systems such as this which are sensitive to phase, they can detect this curvature and this opens the possibility for passive ranging and 3D imaging. Sensitivity to wave-front curvature is generally assumed to be at ranges closer than the Rayleigh distance, which for the DTI imager is around $1.5 \mathrm{~m}$.

If from first principles the phase deviation from spherical to plane waves is calculated across the imager aperture of size $D$ from sources at range $R$, the Eq. 16 results. The examination of the short term (few minutes) phase precision of the DTI imager indicates a figure of $3.2 \times 10^{-4}$ radians. Using this as the phase error in Eq. 16 indicates this imager using its $10 \mathrm{~cm}$ aperture could in principle discriminate the curvature on wave-fronts for sources all the way out to ranges approaching $2 \mathrm{~km}$. 


$$
\Delta \phi=\frac{\pi}{4} \frac{D^{2}}{R \lambda}
$$

Passive ranging or 3D imaging capabilities of the near-field aperture synthesis technique can be assessed by simulation of the DTI imager to image four equi-spaced point sources at a range of $50 \mathrm{~mm}$. The synthetic cross-correlations for this configuration were calculated using Eq. 12. A series of G-matrices were then calculated for the imager at ranges from 49 $\mathrm{mm}$ to $51 \mathrm{~mm}$. These matrices where then inverted using the pseudo inverse with singular value decomposition (SVD) to minimise errors ${ }^{[7]}$. The L-curve for this inversion is given in Figure 9 as a function of baseline index and in the inversion a threshold on the single values of $5 \times 10^{-3}$ was used. The use of the L-curve in minimising the errors is effective in finding solutions (ie the image) where the number of pixels (or unknowns) generated is greater than the number of crosscorrelations (measurements) ${ }^{[8]}$. This is the solution to an underdetermined problem and the SVD and L-curve technique is a way of taking the best solution from many. The images were then calculated by multiplying the inverted G-matrix with the synthetic cross-correlations. The images reconstructed for the sources at the ranges of $49 \mathrm{~mm}, 50 \mathrm{~mm}$ and $51 \mathrm{~mm}$ are shown in Figure 10. The ratio of the amplitude of the four peaks reproduced in the resulting images to the standard deviation of the intensity over the whole image is plotted as a function of range in Figure 9. This simulation indicates the peak of the clearest image appears at a range of $50 \mathrm{~mm}$ with a half power full width of $2 \mathrm{~mm}$. This suggests in this simulation that the range resolution is around $2 \mathrm{~mm}$ which can be compared to the wavelength of $13 \mathrm{~mm}$. This illustrates the power that an interferometric system has in spatial resolution.
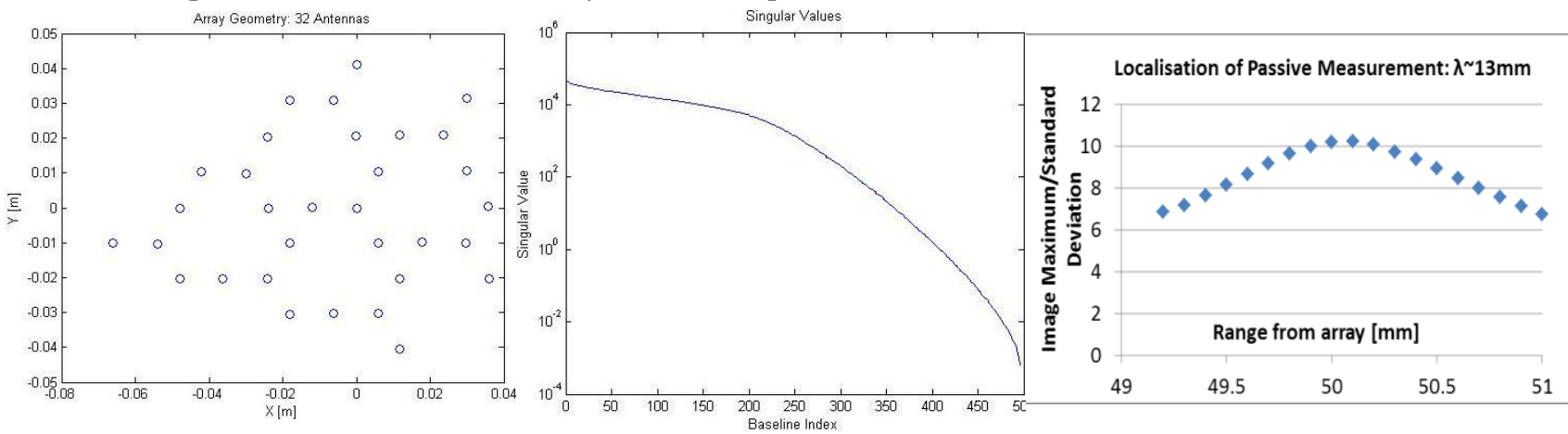

Figure 9: The antenna layout for the DTI imager (left), the singular values of the G-matrix for this antenna layout (centre) and the ratio of peak to standard deviation of the reproduced image of the point sources (right). These suggest in this simulation the range resolution for passive ranging is $2 \mathrm{~mm}$.
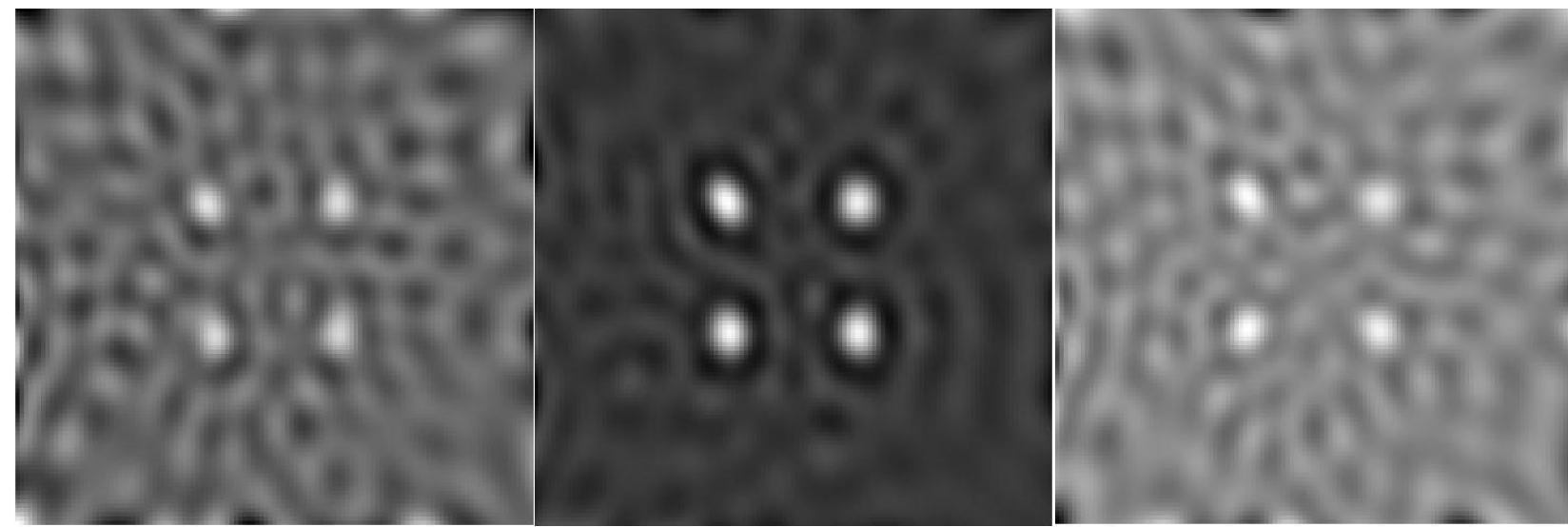

Figure 10: Images reproduced at ranges of $49 \mathrm{~mm}, 50 \mathrm{~mm}$ and $51 \mathrm{~mm}$ from synthetic cross-correlations generated from 4-equispaced point sources at a range of $50 \mathrm{~mm}$ from the antenna array. These images indicate the power of the near-field aperture synthesis technique to localise emission in 3D space.

\subsection{Simulation of Images with larger numbers of pixels}

The concept of aperture synthesis imaging in the near field is proven and the technology to develop follow-on systems with much larger numbers of channels exists. It is now timely to develop simulation tools to illustrate the capabilities of 
imagers in this next evolutionary step. The follow-on imager system should generate images of sufficiently high quality to release funding for prototype development. This next phase imager needs to generate images with tens of thousands of pixels with a spatial resolution of the order of $1 \mathrm{~cm}$ on a person for security screening.

Using the simulation code an array with 111 antennas in random locations was created over a $40 \mathrm{~cm}$ sized aperture and this is illustrated in Figure 11. Using Eq.12 synthetic cross-correlations were generated and this then used with the Fourier transform technique to generate the image of the Vitruvian man. In order that the Fourier transform technique could be used the subject was placed in the far field. The image reproduced is illustrated in Figure 11. The increase in the number of channels is reflected in the improved image quality. In the generation of the cross-correlations, the complexity in the numerics is proportional to $\mathrm{N}_{R}{ }^{2} \mathrm{~N}_{S}$, where $\mathrm{N}_{\mathrm{R}}$ is the number of receiver channels and $\mathrm{N}_{\mathrm{S}}$ is the number of sources in the scene. This means for complex scenes and many receiver channels (necessary to achieve good quality images) the simulation becomes computationally intensive.

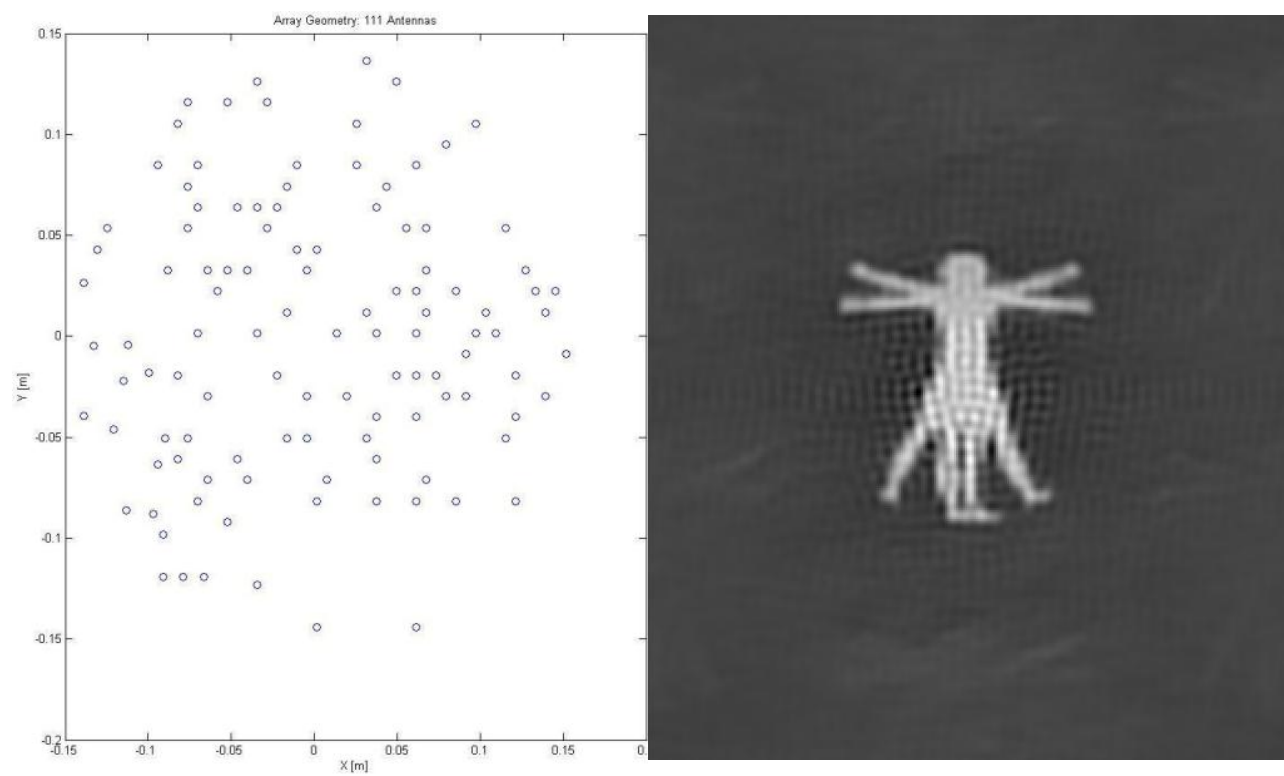

Figure 11: A random array having 111 receiving antennas distributed over a diameter of $40 \mathrm{~cm}$ and the resulting simulated image of the Vitruvian man.

\section{SUMMARY}

Near-field phase effects have been evaluated and it is concluded that for a small aperture synthesis imager with an aperture of $10 \mathrm{~cm}$ phase effects are sufficiently small to enable the Fourier transform technique to create images at a range of $0.5 \mathrm{~m}$, which is within the Rayleigh distance. However, for larger aperture synthesis imagers approaching $0.5 \mathrm{~m}$ in size the phase errors are several hundred degrees and an alternative technique, such as the Gmatrix method has to be used. A scheme for generating synthetic cross-correlations was presented and used effectively to reproduce the effects of aliasing in measured data. The simulation was then extended to show how passive ranging in the near-field was effective at localising the ranges of sources to an accuracy of $2 \mathrm{~mm}$ using radiation having a wavelength of $13 \mathrm{~mm}$. The simulation technique was extended to illustrate images generated from a system with 111 receiver channels.

\section{FUTURE WORK}

Future work will continue with the development of software to efficiently simulate imagery generated with aperture synthesis imaging systems having hundreds of channels. On-going work is looking to minimise the cost of a next phase demonstrator that will employ such numbers of receivers, sampling in single bits at hundreds of $\mathrm{MHz}$ and processing data using systems that incorporate several Field Programmable Gate Arrays.

\section{REFERENCES}

[1] Salmon, N.A. et al, "First video rate imagery from a 32-channel 22-GHz aperture synthesis passive millimetre wave imager", SPIE Europe Security+Defence, Millimetre Wave and Terahertz Sensors and Technology, Prague, September, (2011) 
[2] Salmon, N.A. et al, "Amplitude and intensity interferometry using satellite LNB receivers for innovative and low cost microwave and millimetre wave sensor development", SPIE Europe Security+Defence, Millimetre Wave and Terahertz Sensors and Technology, Edinburgh, September, (2012)

[3] Salmon, N.A. et al, "First imagery generated by near-field real-time aperture synthesis passive millimetre wave imagers at $94 \mathrm{GHz}$ and $183 \mathrm{GHz}$ ", SPIE Europe Security+Defence, Millimetre Wave and Terahertz Sensors and Technology, Toulouse, September, (2010)

[4] Born, M, and Wolf, E., "Principle of optics", Cambridge University Press, 7th Edition, (2003)

[5] Thomson, A., Moran, M., Swenson, G, "Interferometry and Synthesis in Radio Astronomy ", Wiley, (2004)

[6] Tanner, A.B, Swift, C.T., "Calibration of a Synthetic Aperture Radiometer", IEEE Transactions on Geoscience and Remote Sensing, Vol. 31., No. 1, January, (1993)

[7] Moon, T.K. and Stirling, W.C., "Mathematical Methods and Algorithms for Signal Processing", Prentice-Hall, (2000)

[8] Hansen, P.C. "Regularization Tools", A Matlab Package for Analysis and Solution of Discrete Ill-Posed Problems, March, (2008) 